\title{
Depressed Women of Low Socioeconomic Status Have High Numbers of Physician Visits in the Year Before Pregnancy: Implications for Care
}

\author{
Jenny Fairthorne ${ }^{\mathrm{a}, \mathrm{b}, \mathrm{d}}$, Gillian E. Hanley ${ }^{\mathrm{b}, \mathrm{c}}$, Tim F. Oberlander ${ }^{\mathrm{b}}$
}

\begin{abstract}
Background: There is a higher prevalence of depression in women of low socioeconomic status (SES) than other women. Further, previous depression is the best predictor of future depression. Therefore, due to the negative effects of maternal depression on the fetus and subsequent child, particularly in combination with low SES, depression is ideally treated before pregnancy. During the year before pregnancy and by SES, we aimed to assess the odds of a physician visit associated with maternal depression and the mean number of physician visits in women by depressive status.
\end{abstract}

Methods: We used population-based registry data of 243,933 women with 348,273 singleton live births in British Columbia from 1999 2009 and estimated family SES decile using tax-file data. Mixed effects logistic regression, adjusting for maternal age and parity, was used to calculate odds ratios and a two-sided, two-sample test was used to compare proportions. STATA 14 was used for analyses.

Results: Compared to women of middle SES (Decile-6), women of low SES (from Decile-1, Decile-2) had higher odds of more than 20 physician visits whether depressed $(\mathrm{aOR}=1.46$ (95\% CI: $(1.15,1.86)$; $\mathrm{aOR}$ $=1.26$ (95\% CI: $(0.98,1.61))$ or non-depressed $(\mathrm{aOR}=1.26(95 \% \mathrm{CI}$ : $(1.13,1.41)$; aOR $=1.24$ (95\% CI: $(1.11,1.38))$ during the year before pregnancy. During pre-pregnancy, depressed women had more than three times the mean number of physician visits than non-depressed women: $(8.56(8.38,8.73)$ versus $(2.59(2.57,2.61), \mathrm{P}<0.00005$.

Conclusions: Physicians have ample opportunities to assess women of child-bearing age for depression and to refer for appropriate treatment. It is particularly important that physicians pay extra attention to identify depression in those of lower SES who are likely to become pregnant.

Manuscript submitted February 4, 2018, accepted March 16, 2018

aDepartment of Health Sciences, University of York, UK

'British Columbia Children's Hospital Research Institute, University of British Columbia, Vancouver, Canada

'Department of Obstetrics and Gynecology, University of British Columbia, Vancouver, Canada

${ }^{\mathrm{d} C o r r e s p o n d i n g ~ A u t h o r: ~ J e n n y ~ F a i r t h o r n e, ~ D e p a r t m e n t ~ o f ~ H e a l t h ~ S c i e n c e s, ~}$ Seebohm Rowntree Building, University of York, Heslington, York, YO10 5DD, UK. Email: Jenny.Fairthorne@york.ac.uk

doi: https://doi.org/10.14740/jocmr3377w
Further, identifying depression and providing appropriate referral for treatment in all women who are likely to become pregnant, are already pregnant or are caring for children is important. In such a way, the possible negative effects of prenatal and post-partum depression, along with the interactive effects of low SES on the child, might be reduced.

Keywords: Socioeconomic status; Maternal; Depression; Preconception; Physician

\section{Introduction}

Over many years, researchers have consistently reported the higher prevalence of depression in those of lower socioeconomic status (SES). As early as the mid-twentieth century, longitudinal research in Canada found that the prevalence of depression was highest in those of low SES [1]. This finding was replicated using US data from 2002, with the finding that those from poor urban areas had more than twice the odds of depression of those from wealthier areas [2]. More recently, a study published in 2013 [3] assessed around 4,000 German women aged 18 to 79 years using a written questionnaire and interview and found more than three times the odds of current depression in women of low SES compared to those of high SES. The primary reason for conducting the present study stems from the negative effects of maternal depression on maternal well-being and the interactive effects of maternal depression and low SES on infant and child outcomes.

\section{Maternal depression, low SES, infant and child outcomes}

Nearly one in eight women will experience a perinatal depressive episode [4], which has consequences for the well-being of mother and infant. Prenatal depression is associated with increased risks of smoking, alcohol and other drug use [4], premature birth [5] and premature death [5], lower rates of breastfeeding [6], not having a partner [7, 8], domestic violence [9] and suicide ideation [8]. Furthermore, prenatal depression has been correlated with an increased risk of depression in the offspring [10], along with social/emotional [11] and behavioral problems in the child [12]. In the first, a Bristol-based study of more than 2,500 dyads of mother and adolescent child, 
Pearson et al [10] reported that prenatal depression was a risk factor for offspring depression at 18 years and that the risk increased with the severity of the maternal depression. Other inter-generational associations of post-partum depression have been identified in the offspring $[13,14]$. For example, a European longitudinal study [13], with more than 1,000 mothers, examined the relationship of post-partum depression and child behavior at 2, 3 and 6 months after the birth using the Edinburgh Depression Scale. The children of mothers with postpartum depression at any of these times were more likely to have mental issues when older than 8 years [13]. In addition, interactions of post-partum depression and low SES have been described [10, 15]. For example, post-partum depression was only a risk factor for increased depression in the 18 year old offspring of mothers with low education and this risk increased with the severity of the maternal depression [10]. Elsewhere, researchers concluded that post-partum depression, in combination with poverty, affected the development (and particularly the cognitive development) of very young offspring [15]. It follows that the period preceding pregnancy is important in terms of the mental health of the impending mother since a history of depression is the best predictor of depression during pregnancy and the post-partum [9]. As a result, examining the odds of depression by SES during the period preceding pregnancy provides an informed way to identify those at higher risk for depression and earlier treatment.

\section{Equity of access and SES}

One of the main principles of the Canada Health Act [16] is equity of access for all Canadians. Therefore, it is important to discern whether there is equitable access to physician services prior to pregnancy, according to each of socioeconomic and depressive status. Currently, equity of access to primary care for those of lower SES is uncertain and equity according to depressive status remains to be investigated. A research group [17] investigated physician visits in more than 3,500 Canadian adults by telephone survey. By income quintile, compared to persons in the highest quintile, those in the lowest were assessed as 32\% less likely to have had a physician visit or to have contacted a physician in the previous 12 months [17]. In Manitoba, investigators [18] reported that those of lowest ranked neighbourhood income had more physician visits than those who were highest ranked. In another national Canadian study [19], researchers concluded that the odds of a person using primary care were independent of income and added that once contact had been made, those of lower income were more likely to have more than six visits. We found no examination of the use of physician services in women by depressive status during the year before pregnancy (pre-pregnancy). Such results would enable health care providers to ascertain whether certain groups of women with depression need assistance in accessing services.

Compared to a population-based study using registry data, most studies of maternal depression and SES have had a relatively small number of participants. Large numbers increase power and therefore provide more opportunities to identify groups of women at increased risk of depression. Furthermore, our data enabled us to use a 10-level proxy for SES and the ass- ociated opportunity to discern the effect of smaller differences in SES. Although we searched extensively, we found no other study investigating SES and depression in the period preceding pregnancy and no study investigating maternal depression and SES categorized more finely than quintiles. Importantly, for each birth in our study, our retrospective data enabled us to identify the period beginning 12 months prior to the pregnancy and ending with conception. We found that during the year prior to pregnancy, the odds of a hospitalization associated with depression decreased in a linear fashion over increasing deciles of SES with disadvantaged women from Decile-1 at highest risk and the risk tending to reduce with each increase in decile. Using the same birth cohort and during pre-pregnancy, we aimed to determine associations between maternal SES and the odds of: 1) One or more physician visit associated with depression; 2) Physician visits associated with any medical diagnosis (but including a diagnosis of depression), grouped by number of visits, and maternal depressive status. We also aimed to calculate the mean number of physician visits in women by depressive status during pre-pregnancy. Our final aim was to identify trends over SES which were associated with physician visits and maternal depressive status.

We expected that women of lower SES with depression would have a greater overall use of physician services, due to the free access to physicians in British Columbia (BC), the need for treatment for the depression and the association of depression with both low SES [3] and chronic disease [20,21]. Hence, in response to our first aim, we hypothesized that women of lower SES would have higher odds of at least one physician visit associated with depression during pre-pregnancy than those of higher SES. In relation to a trend over increasing SES, we were unsure whether the odds would reduce abruptly in women of a middle SES and remain at a similar lower level as SES increased or whether the odds would reduce in a linear fashion. We found no research which reported such trends. For the same reasons, in response to the second aim, we hypothesized that women of lower SES would have higher odds of larger numbers of physician visits associated with depression during pre-pregnancy than those of higher SES. Again, we were unsure of the type of trend.

\section{Materials and Methods}

\section{Databases}

We gained ethical approval from the Behavioural Research Ethics Board [22] of the University of BC. Population Data $\mathrm{BC}$ then supplied us with validated datasets $[23,24]$ from the Births Registry [25], BC Perinatal Data Registry [26], Medical Services Provision Registry [27], Medical Insurance Registry [28] and the income data from the Census Registry [29].

\section{Inclusion criteria}

Our study comprised women with a child born in BC from 1999 - 2009. Here, we distinguished between women who 
were resident outside $\mathrm{BC}$ for significant portions of the years around the birth of a child and those without consultations by including only women who were registered with the Medical Services Provision Registry for at least 275 days in the year before, the year of the birth and the year after the year of birth of the indexed child.

\section{Explanatory variables and time period}

From tax-file data, we accessed equivalised family annual income data of 1,000 levels for 2002 and 2006. By post-code, household disposable incomes were averaged and ranked, enabling the formation of 1,000 bands [30]. For births before July 1, 2004, we used the 2002 data and otherwise, data from 2006. By combining the lowest 100 bands we formed Decile-1, combining the next 100 bands, we formed Decile-2 and so on, with Decile-10 representing the most advantaged women. As we were investigating trends over SES, a decile measure of SES is preferable to a five-level measure, as the increased number of levels enable any trend to be more visible. Since the risk of maternal depression is related to age and parity [10], we included maternal age and parity at the infant's birth-date as covariates in the adjusted model.

Our variable for maternal age had four levels: less than 20 years, 20 - 29 years, 30 - 39 years and 40 years or older. Parity was measured by a variable with the levels: nulliparous and multiparous. The infant's birth-date and final gestational age were provided by Perinatal Services BC [26] and we used these variables to estimate the date of conception.

\section{Outcome measures}

\section{Physician visit associated with depression}

For each physician visit, there were up to five ICD-9 codes, but mostly, only one. We defined physician visits with a diagnosis associated with depression using the ICD-9 codes: 296.2, 296.3, 300.4, 311, 290.2, 296.0, 296.1, 296.4 - 296.9, 298.0 298.4, 298.8 - 298.9, 300, 309.0 and 309.1 [31].

\section{Depressive status and number of physician visits}

A woman was assessed as depressed if she had at least one physician visit associated with depression. We created a fourlevel variable for all physician visits, regardless of the related diagnostic code, with the levels of: $0-1 ; 2-10 ; 11-20$ and $>$ 20 and assessed the odds of each level in both women with and without depression.

\section{Analyses}

Over the study period, women had varying numbers of births which resulted in a clustering effect. In order to allow for this, we used mixed effects logistic regression to assess the odds of

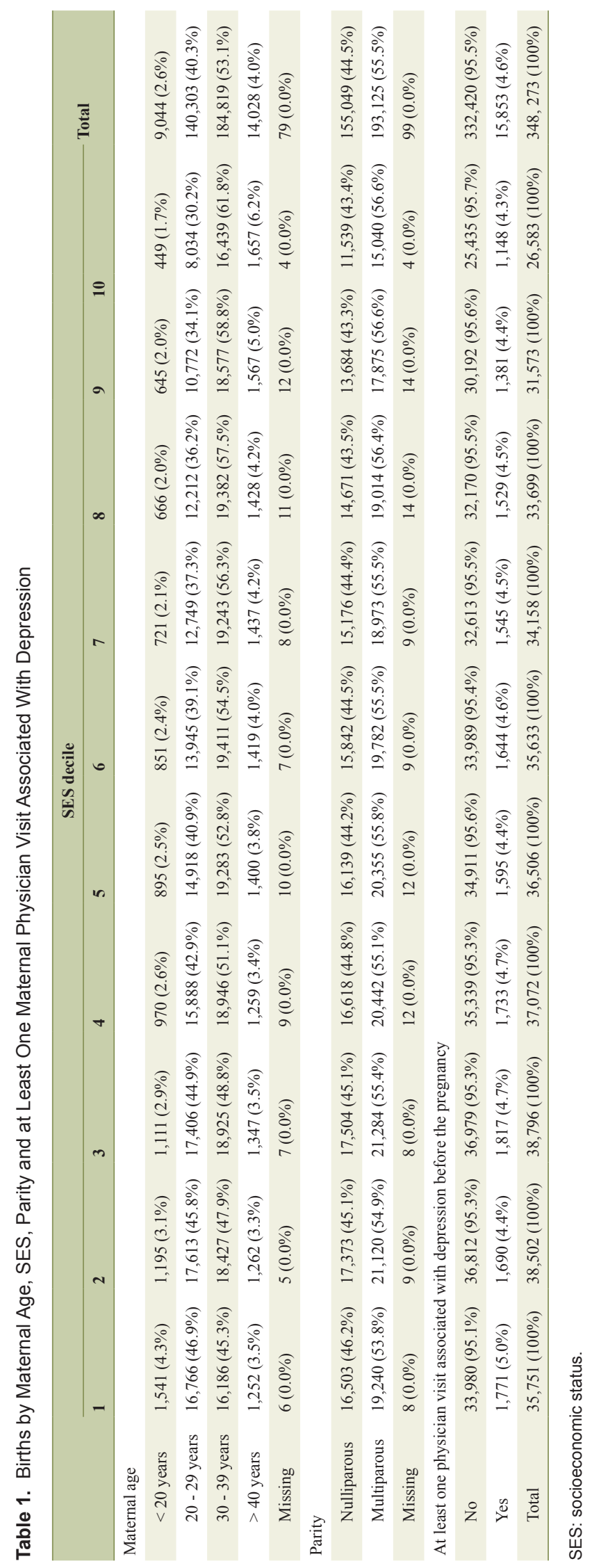


each outcome by birth and SES decile. The comparator group was women from Decile-6 as we wanted to examine the odds ratios (ORs) of both the most disadvantaged mothers (Decile-1) and the most advantaged mothers (Decile-10). For our analyses with the outcome, physician visit associated with depression, we made nine comparisons. Using Bonferroni's approximation for multiple comparisons [32], we adjusted our level of significance by dividing the usual significance level $(0.05)$ by 9 , giving the adjusted level of 0.0056. Similarly, for the second outcome, number of physician visits, we made 27 comparisons in each of women with and without depression which led to a significance level of $0.0019(0.05 / 27)$. Unadjusted odds ratios (uOR) and adjusted odds ratios (aOR) are tabulated but we report only significant aORs. We also investigated the existence of a linear trend for each outcome with SES, again using mixed effects logistic regression. To assess whether depressive status impacted the number of physician services, we performed a two-sample t-test for samples with unequal variance [33]. Finally, to compare proportions of interest relating to SES and demographic traits, we used a two-sided, two-sample test of proportions [32] accessed by using the "prtesti" command from STATA [34]. STATA14 was used for all analyses.

\section{Results}

In this study there were 243,393 women with 348,273 births (Table 1) and 995,895 physician visits. In about $4.6 \%$ of pregnancies, women were depressed (Table 1) and 5.0\% of these pregnancies were associated with Decile-1 and 4.3\% with Decile-10 (Table 1). The difference between these extreme deciles was not significant $(\mathrm{P}=0.4286)$.

\section{At least one physician visits associated with depression}

Odds of physician visits associated with depression during pre-pregnancy are in Table 2. Over increasing SES, there was a negative linear trend $(\mathrm{P}=0.034)$.

\section{Number of physician visits by depressive status}

Women without depression from Decile-1 were about $26 \%$ more likely to have more than 20 physician visits $(\mathrm{aOR}=1.26$ (CI: $1.13,1.41) \mathrm{P}<0.0005)$ and women from Decile-2 were about $24 \%$ more likely to have more than 20 physician visits $(\mathrm{aOR}=1.24(\mathrm{CI}: 1.11,1.38) \mathrm{P}<0.0005)($ Table 3$)$. In women with depression with more than 20 visits, there was a significant associated negative linear trend over increasing SES ( $\mathrm{P}=$ 0.021 ). Distribution of physician visits by depressive status is in Supplementary 1 (Supplementary Table 1, www.jocmr.org).

\section{Depressive status and mean number of physician visits}

The mean number of physician visits was 2.59 (95\% CI: 2.57 , 2.61) for women without depression and 8.56 (95\% CI: 8.38 ,
Table 2. Odds* of at Least One Physician Visit Associated With Depression During Pre-Pregnancy by SES

\begin{tabular}{|c|c|c|c|}
\hline SES decile & Model & No visit & Odds ratio \\
\hline \multirow[t]{2}{*}{1} & $\mathrm{uOR}$ & $\ldots$ & $1.13(1.05,1.22)^{* *}$ \\
\hline & $\mathrm{aOR}$ & & $1.10(1.02,1.19)$ \\
\hline \multirow[t]{2}{*}{2} & $\mathrm{uOR}$ & $\ldots$ & $0.97(0.90,1.05)$ \\
\hline & $\mathrm{aOR}$ & & $0.97(0.89,1.04)$ \\
\hline \multirow[t]{2}{*}{3} & $\mathrm{uOR}$ & $\ldots$ & $1.04(0.97,1.12)$ \\
\hline & $\mathrm{aOR}$ & & $1.03(0.96,1.11)$ \\
\hline \multirow[t]{2}{*}{4} & uOR & $\cdots$ & $1.05(0.98,1.14)$ \\
\hline & $\mathrm{aOR}$ & & $1.05(0.97,1.13)$ \\
\hline \multirow[t]{2}{*}{5} & $\mathrm{uOR}$ & $\ldots$ & $0.96(0.89,1.04)$ \\
\hline & $\mathrm{aOR}$ & & $0.97(0.89,1.04)$ \\
\hline 6 & Comparator group & & \\
\hline \multirow[t]{2}{*}{7} & $\mathrm{uOR}$ & $\cdots$ & $1.00(0.93,1.09)$ \\
\hline & $\mathrm{aOR}$ & & $1.01(0.93,1.09)$ \\
\hline \multirow[t]{2}{*}{8} & uOR & $\cdots$ & $1.04(0.96,1.12)$ \\
\hline & $\mathrm{aOR}$ & & $1.05(0.97,1.13)$ \\
\hline \multirow[t]{2}{*}{9} & $\mathrm{uOR}$ & $\ldots$ & $1.00(0.93,1.08)$ \\
\hline & $\mathrm{aOR}$ & & $1.01(0.94,1.10)$ \\
\hline \multirow[t]{2}{*}{10} & uOR & $\cdots$ & $0.98(0.90,1.07)$ \\
\hline & $\mathrm{aOR}$ & & $0.99(0.91,1.08)$ \\
\hline
\end{tabular}

SES: socioeconomic status; uOR; unadjusted odds ratio; aOR; adjusted odds ratio; No visit; No physician visit associated with depression. ${ }^{* *} \mathrm{P}<0.0056$ (lowered from 0.05 in accordance with Bonferroni's approximation). *Age at the birth of the child and parity are included in the multivariate model.

8.73) for women with depression. The difference between the two means was significant $(\mathrm{P}<0.00005)$.

\section{Discussion}

Using population-based data from BC, we found that women of the lowest SES had highest odds of a physician visit associated with depression during pre-pregnancy. We also found that women with depression had significantly more physician visits with any diagnosis. Thus, there was no evidence of inequity of access to physicians in BC, by either SES or depressive status.

In line with our first hypothesis, during the pre-pregnancy period, women from the lowest income decile were significantly more likely to have at least one physician visit associated with depression than women from Decile-6. However, after adjustment for age and parity, the odds were no longer significant. This could reflect the impact of maternal age and parity, or an interaction between the two, on the odds of having a physician visit associated with depression. Overall, these findings reflect the established association of depression and low SES in Western society [1-3].

In accord with our second hypothesis, women of lowest SES, both with and without depression, had highest odds of 
Table 3. Odds* of Various Numbers of Physician Visits in Women by Depressive Status and SES During Pre-Pregnancy

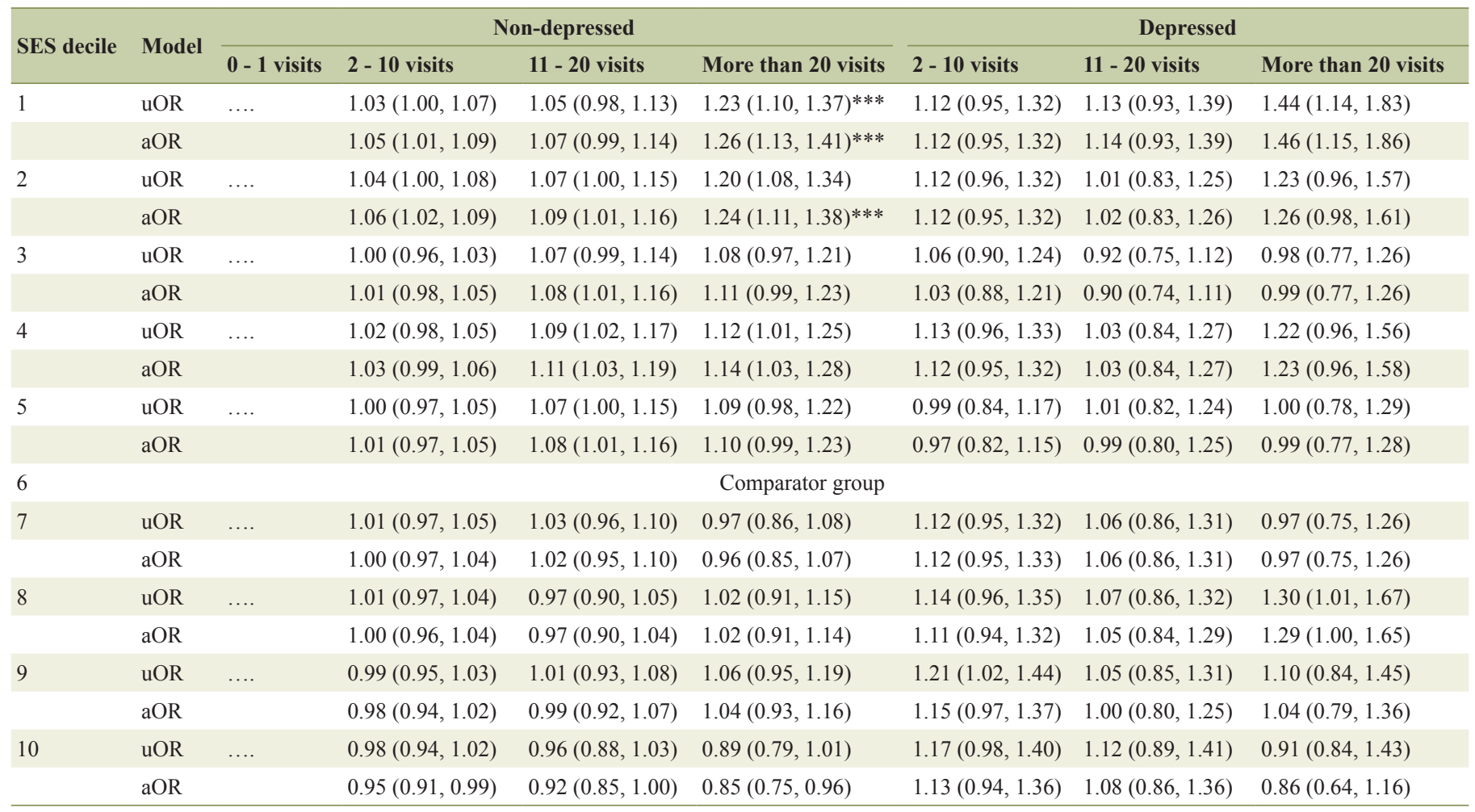

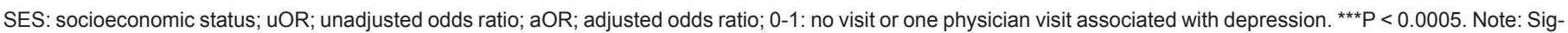

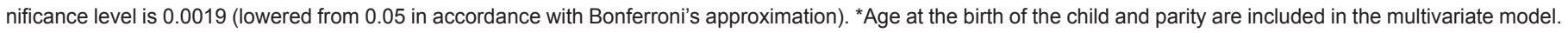

large numbers of physician visits during pre-pregnancy. The odds reduced in a linear fashion over increasing SES, though for women without depression the trend did not reach significance. This indicates a negative gradient over increasing SES, with increasing disposable family income reducing the likelihood of a woman consulting a physician during the prepregnancy. It also provides further evidence of a negative gradient over increasing SES for depression in women during the pre-pregnancy period. There are strong associations between low SES and chronic conditions such as chronic obstructive pulmonary disease and type 2 diabetes [20, 21]. Such associations would be likely to contribute to increased physician visits in low SES women without depression. This study may be the first to find that the rate of services associated with depression during pre-pregnancy are not only highest in the most disadvantaged women but that risk reduces in a linear fashion with improving economic circumstances. Our proxy for SES was disposable income and it follows that women in the lowest SES decile are most likely to suffer from depression arising from a difficulty in accessing the necessities of life. In addition, domestic violence is correlated with both prenatal depression and lower SES [9]. In BC, there is no evidence that women of lower SES have difficulty utilizing physician services in response to their health needs. The results of Dunlop et al [19] support ours, although timing in relation to pregnancy and depressive status were not addressed. In this study [19], compared to Quintile-5 (most advantaged), women from the Quintile-1 and Quintile-2 had 30\% and 21\% higher odds of more than six physician visits [19]. People of lower SES tend to have poorer general health [35]. From this perspective, we suggest that the increased use of physician services in those of lower SES, regardless of depressive status, might be due to their poorer general health.

Our third hypothesis was validated since women with depression had more than three times the number of physician visits (8.6 versus 2.6 ) of women without depression. This could be explained in terms of visits to address their depressive status, which would include visits for assessment, prescriptions and referral(s) to specialists. In addition, women with depression would be more likely to have increased visits associated with other diagnoses due to their higher expected number of comorbidities [20,21]. Overall, the higher use of physician services in women with depression suggests that they have no difficulty in visiting a physician. This information, combined with our findings that women of lowest SES have increased odds of a physician visit associated with depression and increased odds of more than 20 visits, regardless of depressive status, suggests that there is no inequity of access by either SES or depressive status in BC.

\section{Strength and limitations}

A strength of this study was the large number of women, enabled by our use of registry data. This increased power allowed us to access smaller differences between groups than previous studies. Our proxies for depression, a physician visit with a 
diagnosis associated with depression and number of physician visits, were objective and did not rely on maternal or family recall. Our 10-level proxy for SES enabled us to examine depression across a social gradient. This is the first study to find increased odds of physician visits associated with depression in women of lower SES during the 12 months before pregnancy. It is also the first to describe increased odds of greater numbers of physician visits in women during the 12 months before pregnancy, regardless of depressive status.

We estimated maternal SES using equivalised family income by post-code which is a less accurate measure than equivalised income by household [36]. Another limitation was that we were unable to include other dimensions of SES such as education and occupation in this proxy. Also, SES information was often unavailable for the child's birth year. If a woman's income changed considerably between the child's birth year and either 2002 or 2006, this would likely have resulted in an incorrect measure of SES. Also, we had no information on mother's race-ethnicity. If available, this could have been included as a covariate in our adjusted model since some racialethnic groups have increased protection from the effects of low SES on depression [37].

\section{Conclusions}

During pre-pregnancy, women of lowest SES had the highest risk of being depressed and the highest risk of the greatest number of physician visits. Due to the potential negative, interactive effects of low SES and maternal depression, it is crucial that women of child-bearing age and of low SES with depression are given adequate opportunities for referral and treatment as soon as possible in relation to a pregnancy. Therefore physicians should pay extra attention to identify depression in these women who are likely to become pregnant, who are already pregnant or who are caring for children, as early as possible in order to facilitate treatment. Further, the importance of identifying and treating women with depression, particularly those of low SES, might be incorporated into any emergent national guidelines for preconception care [38]. The higher use of physician services in women of low SES with depression during pre-pregnancy suggests that physician referral of women at high risk to appropriate treatment programs might be an effective way for them to access such care. In such a way, the adverse, interactive and intergenerational effects of maternal depression during pregnancy and the post-partum, might be reduced.

\section{Conflict of Interest}

No author declared a conflict of interest related to this manuscript.

\section{References}

1. Hirschfeld RM, Cross CK. Epidemiology of affective disorders. Arch Gen Psychiatry. 1982;39(1):35-46.

2. Galea S, Ahern J, Nandi A, Tracy M, Beard J, Vlahov D.
Urban neighborhood poverty and the incidence of depression in a population-based cohort study. Ann Epidemiol. 2007;17(3):171-179.

3. Busch MA, Maske UE, Ryl L, Schlack R, Hapke U. Prevalence of depressive symptoms and diagnosed depression among adults in Germany: results of the German Health Interview and Examination Survey for Adults (DEGS1). Bundesgesundheitsblatt Gesundheitsforschung Gesundheitsschutz. 2013;56(5-6):733-739.

4. Meltzer-Brody S. New insights into perinatal depression: pathogenesis and treatment during pregnancy and postpartum. Dialogues Clin Neurosci. 2011;13(1):89-100.

5. Gentile S. Untreated depression during pregnancy: Shortand long-term effects in offspring. A systematic review. Neuroscience. 2017;342:154-166.

6. Davalos DB, Yadon CA, Tregellas HC. Untreated prenatal maternal depression and the potential risks to offspring: a review. Arch Womens Ment Health. 2012;15(1):1-14.

7. Bodecs T, Szilagyi E, Cholnoky P, Sandor J, Gonda X, Rihmer Z, Horvath B. Prevalence and psychosocial background of anxiety and depression emerging during the first trimester of pregnancy: data from a Hungarian populationbased sample. Psychiatr Danub. 2013;25(4):352-358.

8. Gavin AR, Melville JL, Rue T, Guo Y, Dina KT, Katon WJ. Racial differences in the prevalence of antenatal depression. Gen Hosp Psychiatry. 2011;33(2):87-93.

9. Lancaster CA, Gold KJ, Flynn HA, Yoo H, Marcus SM, Davis MM. Risk factors for depressive symptoms during pregnancy: a systematic review. Am J Obstet Gynecol. 2010;202(1):5-14.

10. Pearson RM, Evans J, Kounali D, Lewis G, Heron J, Ramchandani PG, O'Connor TG, et al. Maternal depression during pregnancy and the postnatal period: risks and possible mechanisms for offspring depression at age 18 years. JAMA Psychiatry. 2013;70(12):1312-1319.

11. Junge C, Garthus-Niegel S, Slinning K, Polte C, Simonsen TB, Eberhard-Gran M. The impact of perinatal depression on children's social-emotional development: a longitudinal study. Matern Child Health J. 2017;21(3):607-615.

12. Leis JA, Heron J, Stuart EA, Mendelson T. Associations between maternal mental health and child emotional and behavioral problems: does prenatal mental health matter? J Abnorm Child Psychol. 2014;42(1):161-171.

13. Closa-Monasterolo R, Gispert-Llaurado M, Canals J, Luque V, Zaragoza-Jordana M, Koletzko B, et al. The effect of postpartum depression and current mental health problems of the mother on child behaviour at eight years. Maternal and Child Health Journal. 2017;21(7):1-10.

14. Avan B, Richter LM, Ramchandani PG, Norris SA, Stein A. Maternal postnatal depression and children's growth and behaviour during the early years of life: exploring the interaction between physical and mental health. Arch Dis Child. 2010;95(9):690-695.

15. Petterson SM, Albers AB. Effects of poverty and maternal depression on early child development. Child Dev. 2001;72(6):1794-1813.

16. Hutchison B, Levesque JF, Strumpf E, Coyle N. Primary health care in Canada: systems in motion. Milbank Q. 2011;89(2):256-288. 
17. Blackwell DL, Martinez ME, Gentleman JF, Sanmartin C, Berthelot JM. Socioeconomic status and utilization of health care services in Canada and the United States: findings from a binational health survey. Med Care. 2009;47(11):1136-1146.

18. Roos LL, Walld R, Uhanova J, Bond R. Physician visits, hospitalizations, and socioeconomic status: ambulatory care sensitive conditions in a Canadian setting. Health Serv Res. 2005;40(4):1167-1185.

19. Dunlop S, Coyte PC, McIsaac W. Socio-economic status and the utilisation of physicians' services: results from the Canadian National Population Health Survey. Soc Sci Med. 2000;51(1):123-133.

20. Gershon AS, Warner L, Cascagnette P, Victor JC, To T. Lifetime risk of developing chronic obstructive pulmonary disease: a longitudinal population study. Lancet. 2011;378(9795):991-996.

21. Stringhini S, Batty GD, Bovet P, Shipley MJ, Marmot MG, Kumari M, Tabak AG, et al. Association of lifecourse socioeconomic status with chronic inflammation and type 2 diabetes risk: the Whitehall II prospective cohort study. PLoS Med. 2013;10(7):e1001479.

22. University of British Columbia. Behavioural Research Ethics 2016 [2017 Sept 15]. Available from: https://ethics.research.ubc.ca/behavioural-research-ethics.

23. Hu W. Diagnostic codes in MSP claim data11996. Available from: http://secure.cihi.ca/cihiweb/dispPage.jsp?cw page $=$ GR 27_E\&cw topic $=27$.

24. Frosst G, $\overline{\text { Hutcheon }} \overline{\mathrm{J}}$, Joseph K, Kinniburgh B, Johnson C, Lee L. Validating the British Columbia Perinatal Data Registry: a chart re-abstraction study. BMC Pregnancy and Childbirth. 2015;15(1):123.

25. Vital Statistics Births. V2 [Internet]. Population Data BC; Data extract. 2014. Available from: http://www.popdata. bc.ca/data.

26. Perinatal Services BC. British Columbia Perinatal Data Registry: Population Data BC; Data Extract 2015. Available from: http://www.perinatalservicesbc.ca/health-professionals/data-surveillance/perinatal-data-registry.

27. British Columbia Ministry of Health. Medical Services Plan (MSP) Payment Information File. V2: Population Data BC; 2015. Data Extract. MOH (2015). Available from: https://www.popdata.bc.ca/data/internal/health/msp.

28. BC Ministry of Health. Consolidation File 2011 (MSP Registration \& Premium Billing): Data Extract MOH: Population Data BC; 2013. Available from: http://www. popdata.bc.ca/data.

29. Statistics Canada. Small area and administrative data division: income band data. 2007. Available from: https:// www.popdata.bc.ca/data/internal/demographic/incomeband.

30. Population Data BC. Income band data 2017. Available from: https://www.popdata.bc.ca/data/internal/demographic/incomeband.

31. Oberlander TF, Warburton W, Misri S, Aghajanian J, Hertzman C. Neonatal outcomes after prenatal exposure to selective serotonin reuptake inhibitor antidepressants and maternal depression using population-based linked health data. Archives of General Psychiatry [Internet]. 2006; 63(8):898-906.

32. Woodward M. Epidemiology: study design and data analysis. Sydney, NSW: CRC press; 2013.

33. Institute for Digital Research and Education. Independent sample T-test assuming unequal variances Los Angeles: University of California; 2017 [2017 April 2]. Available from: http://stats.idre.ucla.edu/stata/output/t-test/.

34. Stata Corporation. STATA 14 statistical software College Station, TX22015.Available from: https://www.stata.com/ stata14/.

35. Schafer I, Hansen H, Schon G, Hofels S, Altiner A, Dahlhaus A, Gensichen J, et al. The influence of age, gender and socio-economic status on multimorbidity patterns in primary care. First results from the multicare cohort study. BMC Health Serv Res. 2012;12:89.

36. Hanley GE, Morgan S. On the validity of area-based income measures to proxy household income. BMC Health Services Research [Internet]. 2008; 8(1):79.

37. Williams MT, Chapman LK, Wong J, Turkheimer E. The role of ethnic identity in symptoms of anxiety and depression in African Americans. Psychiatry Res. 2012;199(1):31-36.

38. Bialystok L, Poole N, Greaves L. Preconception care: call for national guidelines. Can Fam Physician. 2013;59(10):10371039, e1435-1037. 